\title{
Medición de la cristalización electoral de un movimiento de protesta: de la indignación al voto
}

\author{
Measurement of the electoral crystallization of a social \\ movement: from indignation to vote
}

\author{
JOSEP LOBERA \\ Universidad Autónoma de Madrid \\ Jesús Rogero-García \\ Universidad Autónoma de Madrid \\ jesus.rogero@uam.es (ESPAÑA)
}

Recibido: 27.092016
Aceptado: 14.09 .2017

\section{RESUMEN}

Nuestro trabajo trata de aportar nuevas aproximaciones metodológicas y conceptuales al análisis del impacto electoral de movimientos sociales y de protesta. Dos campos de estudio, el de los movimientos sociales y el de nuevos partidos políticos, parecen haber dejado un hueco analítico escasamente tratado entre los dos: la conexión entre el auge de movimientos sociales y la posterior aparición de nuevos partidos políticos. Concretamente, nos interesa indagar cómo se comportan electoralmente las bases de un movimiento de protesta ante la aparición de un nuevo partido que, teóricamente, recoge las demandas centrales del movimiento. Analizamos el paso del apoyo al movimiento $15 \mathrm{M}$ (conocido también como los indignados) al apoyo electoral a Podemos tras sus primeros meses de existencia. Para ello introducimos el cálculo de un índice de cristalización electoral para diferentes grupos sociales, lo que nos permite contribuir a la caracterización de la emergencia del nuevo partido. Los datos apuntan a que el voto a Podemos en las elecciones europeas de 2014 puede entenderse como a una extensión de la protesta en el campo electoral. Esta continuidad con la protesta parece caracterizar la aparición del nuevo partido, tanto en la base social de su primer electorado como en lo que se refiere a su aspecto narrativosimbólico. Su primera base electoral no respondería tanto a un voto de protesta - en el sentido planteado por Van der Eijk- sino, más bien, a un voto de la 
protesta, consiguiendo captar electoralmente a aquellos simpatizantes del $15 \mathrm{M}$ más identificados con sus principales reivindicaciones. Finalmente, contrastando los resultados con otros estudios, apuntamos que la crisis política y de confianza institucional tendría un efecto asimétrico en la dinámica electoral a lo largo del arco ideológico, de un modo mucho más intenso de lo que ocurre en el apoyo a los movimientos de protesta. Así, la ideología se presenta como el principal regulador del impacto electoral de la insatisfacción política entre la ciudadanía.

\section{PALABRAS CLAVE}

Nuevos partidos políticos; Movimientos sociales; Institucionalización; 15M; Podemos.

\section{ABSTRACT}

This article examines the electoral impact of social protest movements, providing new methodological and conceptual approaches to the subject. Two fields of study, social movements and new political parties, seem to have left a sparsely treated gap between the two: the connection between the rise of social movements and the subsequent emergence of new political parties. Specifically, we are interested in investigating how the foundations of a protest movement electorally behave at the emergence of a new party -one that theoretically reflects the central demands of the movement. We analyze how the support to the $15 \mathrm{M}$ movement (also known as the Indignants) has evolved into electoral support to Podemos after its first months of existence. To do this, we compare the social profiles of $15 \mathrm{M}$ supporters and Podemos voters through the introduction of an electoral crystallization indication. The results suggest that Podemos vote in the 2014 European elections can be seen as an extension of the protests in the electoral field. This continuity with the protest seems to characterize the appearance of the new party, both in the social base of its first electorate as in regard to its narrative-symbolic aspect. Further, its first electoral base did not respond so much to a 'protest vote' -in the sense proposed by Van der Eijk- but rather to a 'vote of the protest', gaining electoral support from those more identified with the $15 \mathrm{M}$ demands. Finally, comparing the results with other studies, we noted that the political and institutional confidence crisis has an asymmetric effect on the electoral dynamics along the ideological spectrum, a much more intense one than observed on the support to the protest movements. Thus, the ideology is presented as the main regulator of the electoral impact of political dissatisfaction among citizens. 


\section{KEY WORDS}

New political parties; Social movements; institutionalization; 15M; Podemos.

\section{INTRODUCCIÓN ${ }^{1}$ :}

La relación entre movimientos sociales y partidos políticos ha sido intensa durante los últimos doscientos años (Tilly, 2004). Partidos, grupos de interés y movimientos sociales son los principales vehículos de articulación e intermediación del interés político (Kitschelt, 2006). A pesar de que existe numerosa literatura sobre movimientos sociales y más aún sobre partidos políticos, el paso de movimientos sociales y de protesta a formaciones políticas ha sido escasamente tratado. En concreto, no existen estudios cuantitativos que midan el trasvase de apoyos de movimientos sociales o de protesta a nuevas alternativas electorales.

La relación entre el 15M y Podemos en España ha suscitado un rápido interés y ha sido tratada en diversos estudios recientes (entre otros, Andretta y Della Porta, 2015; Calvo y Álvarez, 2015; Calle, 2015; Martín, 2015; Romanos y Sádaba, 2016; Tormeya y Feenstra et al., 2017). Este interés, en gran medida, se debe a que la relación entre el 15M y Podemos puede ser considerada como un caso paradigmático para aportar luz a este tipo de procesos, tanto por las magnitudes de movilización como por la rapidez de la emergencia de ambos casos.

La crisis política ha sido señalada como una de las causas, si no la principal, de la eclosión de las protestas de los indignados (Sampedro y Lobera, 2014; Muro y Vidal, 2016) así como del voto a Podemos (Orriols y Cordero, 2016) en las elecciones generales de 2015. La observación del desarrollo de esa crisis política entre la opinión pública, desde 2008 a 2016, nos lleva a argumentar que existe una cierta continuidad en los procesos de erosión del apoyo político, el desarrollo del ciclo de protestas, la emergencia de nuevos partidos en 2014 y el fin del sistema bipartidista en 2015. El cómo se produce esta continuidad, especialmente el paso del apoyo a la protesta al voto a Podemos, es objeto de este trabajo. Entre otras cosas, en esta continuidad veremos que los factores que condicionan el voto a la nueva formación no coinciden con los que condicionan la simpatía por el movimiento de protesta.

El auge de los movimientos sociales durante 2011 en España coincide con un momento de especial debilidad de la política partidista para generar confianza en los ciudadanos (Muro y Vidal, 2016; Flesher Fominaya, 2014; Lobera y Ferrándiz, 2013), una crisis política similar a la observada en otros países del Sur de

1 Este trabajo es un desarrollo de las siguientes comunicaciones: Josep Lobera y Jesús Rogero-García (2016). "La medición de la cristalización electoral del 15-M en Podemos", XII Congreso Español de Sociología, FES, Gijón; Josep Lobera y Jesús Rogero García (2015). "The Podemos emergence: Electoral crystallization of the 15-M support?', Eighth CIS Summer Seminar on Sociological and Political Research, RCC-Harvard University, Cambridge, MA. 
Europa (Bosco y Verney, 2012). Entre sus mensajes centrales estaban "No nos representan", en referencia a la clase política, y "No les votes", la propuesta de no votar al PSOE ni al PP — junto con CiU en Cataluña y PNV en País Vascoy que se optara por algún partido minoritario o bien por el voto nulo. Cualquier tipo de protesta social puede interpretarse, como señala Kasse (2007), como una forma no institucionalizada de implicación política. No es extraño, por tanto, que un movimiento de protesta pueda $-\mathrm{y}$ desee- extenderse hacia la esfera de la política representativa. Esta extensión puede tener múltiples facetas, desde la influencia en la regeneración de partidos ya existentes, pasando por la promoción de ciertos patrones de voto, hasta la creación de nuevas formaciones políticas.

Las movilizaciones del $15 \mathrm{M}$ no tuvieron, de forma inmediata, más que un pequeño efecto electoral en las elecciones convocadas en 2011 -que consistió en una erosión del bipartito y una pequeña propensión a votar a dos partidos minoritarios, IU y UPyD (Anduiza et al., 2012; Jiménez, 2011), particularmente en la Comunidad de Madrid. Sin embargo, la emergencia de nuevos partidos tras la finalización del ciclo de movilizaciones, primero en las elecciones al Parlamento Europeo (PE) de 2014 y su consolidación posterior en las locales y regionales de 2015 y en las elecciones generales de ese mismo año, supuso el paso de un sistema partidista a uno multipartidista (Orriols y Cordero, 2016; Rodón y Hierro, 2016; Cordero y Montero, 2015).

Desde su inicio, los movimientos de protesta tuvieron, explícitamente, la intención de influir en el campo electoral (\#nolesvotes, \#PPSOE, entre otras iniciativas). Tras la finalización del ciclo de protestas, una parte importante del activismo del $15 \mathrm{M}$ vio en los nuevos partidos "una nueva táctica del movimiento" (Romanos y Sádaba, 2015) y se intensificaron los intentos por trasladar a la política institucional el extenso apoyo ciudadano que recibieron. Las nuevas propuestas políticas surgidas en el marco simbólico y comunicativo del 15M (Ahora tú decides, Alternativas desde Abajo, Confluencia, Red Ciudadana Partido X, Podemos, entre otros) tuvieron como prioridad captar votantes de ese nuevo espacio simbólico-comunicativo. Será Podemos el primero en obtener representación política en este espacio comunicativo-simbólico, con cinco eurodiputados en las elecciones al PE de 2014, apenas cuatro meses tras su presentación oficial.

A pesar de la importancia de ambos procesos, la principal dificultad para analizar cuantitativamente el paso del apoyo al movimiento de protesta a la emergencia de la nueva base electoral de Podemos reside en la inexistencia de encuestas en 2014 que, al mismo tiempo, pregunten por el apoyo al 15M y la intención o recuerdo de voto. Para reducir las limitaciones que impone esa dificultad, proponemos el cálculo de un índice de cristalización electoral para diferentes grupos sociales mediante el cual poder identificar el grado en el que distintos grupos sociales han traducido su apoyo al 15M en 2011 a apoyo electoral a Podemos en 2014. Esta cuantificación nos aporta una información relevante para constatar o rebatir algunas hipótesis sobre cómo se produce ese proceso.

Nuestra intención, así, es introducir un elemento más en la literatura académica que, junto con otros, permitan caracterizar cómo ha tenido lugar la emergencia de esa nueva formación política. En una primera parte de nuestro 
trabajo, repasamos las principales aportaciones teóricas de lo que llamamos "cristalización electoral" de movimientos sociales o de protesta (Lobera, 2015): el proceso por el cual una parte de los simpatizantes de estos movimientos traslada su apoyo electoral a nuevas alternativas políticas tras un ciclo de protestas particularmente intenso. Aquí, abordamos principalmente las teorías de la emergencia de los partidos verdes desde los movimientos ecologistas en Alemania, de los partidos-movimiento indigenistas de América Latina y, más recientemente de nuevos partidos de izquierda europeos, como Syriza en Grecia. En una segunda parte, planteamos la metodología utilizada y, como una aportación original de este trabajo, el cálculo de un índice de cristalización electoral (Ic). En tercer lugar, exponemos los resultados que obtenemos a partir de tres modelos de regresión logística bivariada y las diferencias de cristalización electoral entre grupos sociales. Finalmente, relacionamos los resultados obtenidos con las teorías de la emergencia de los partidos -desarrolladas en la primera parte del trabajo- y discutimos la emergencia de Podemos a partir de las diferencias observadas entre grupos sociales en la cristalización electoral del apoyo al movimiento de protesta.

\section{MARCO TEÓRICO}

¿Qué factores favorecen la aparición de nuevos partidos? Se han realizado numerosas investigaciones sobre la emergencia de nuevos partidos, pero los casos más estudiados por la literatura responden a realidades y dinámicas claramente diferenciadas de nuestro caso de estudio. Algunos casos, sin embargo, pueden aportar un marco teórico de referencia para nuestro análisis: el estudio de la emergencia de los partidos verdes desde los movimientos ecologistas, de los partidos-movimiento indigenistas de América Latina, así como de Syriza en Grecia.

Diversas teorías han tratado de explicar por qué los partidos verdes fueron capaces de entrar en los sistemas políticos y en muchas democracias occidentales logran presencia parlamentaria sostenida. Uno de los más estudiados ha sido el caso de Die Grünen, el partido verde alemán, debido a su relevancia en la política alemana a partir de 1983 y su entrada en un gobierno de coalición con el SPD en 1998 y 2002. Ronald Inglehart (1995; 1997) proporciona una explicación cultural -posiblemente la más conocida y controvertida- a partir de su teoría de la emergencia de los valores postmaterialistas, mientras que Ferdinand Müller-Rommel (1998; 2002) y Herbert Kitschel (1989), entre otros, adoptan un enfoque estructural, centrado en los factores como el sistema electoral, el entorno económico y la competición electoral.

La emergencia de nuevos partidos en América Latina ha sido, asimismo, objeto de estudio en las últimas décadas y está vinculada con la crisis de los partidos tradicionales en esta región (Alcántara y Freidenberg, 2001; Canton, 1995; Sánchez, 2008). Los estudios de Roberts (1998) y Van Cott (2005) han analizado las alianzas entre los partidos de izquierda y los movimientos sociales. 
Roberts (1998) muestra cómo en Perú y Chile los partidos de izquierda ofrecieron la idea de "profundización de la democracia" como medio para llegar a grupos populares no representados. Por su parte, en su comparación sistemática de los movimientos y los partidos indigenistas en seis países -Ecuador, Bolivia, Colombia, Venezuela, Perú y Argentina-, Van Cott (2005) señala como factores cruciales el acceso a nuevos derechos constitucionales, la fortaleza y unidad de los movimientos sociales indígenas, su difusión por todo el país, y la participación indígena en el proceso de reforma constitucional. En otro contexto, el Partido de los Trabajadores (PT) en Brasil presentó características similares (Sousa Santos, 2014), al buscar dar voz política a las clases populares que hasta entonces no habían visto reflejadas sus demandas en los viejos partidos políticos.

Desde una perspectiva comparada, Irene Martín (2015) señala algunos aspectos organizativos para el estudio de los nuevos partidos surgidos de movimientos sociales en el sur de Europa. Plantea dos tipos: en primer lugar, aquel en el que el origen del partido está en la transformación o unión de movimientos sociales $-\mathrm{y}$, por tanto, la continuidad de aspectos organizativos de estos resulta evidente- $\mathrm{y}$, en segundo lugar, cuando la relación del partido con los movimientos tiene que ver con la imitación o incorporación de aspectos organizativos de los últimos por parte del primero. Al primer modelo, se ajustan tanto el MAS (Bolivia) como las Candidaturas de Unidad Popular surgidas en 2015 en España, como Ahora Madrid o En Comú Podem, mientras que Podemos y Syriza (Grecia) se ajustarían más al segundo modelo (íbid.).

En el caso de Syriza, su confluencia con otros grupos, partidos y movimientos ha sido identificada como una de las principales claves de su éxito electoral (Tsakatika, 2015; Martín, 2015). ${ }^{2}$ La estrategia de Podemos, en cambio, ha sido de una menor confluencia con otras organizaciones y movimientos sociales $-\mathrm{a}$ pesar de que esta ha ido en aumento en las distintas convocatorias electorales desde 2014-. La vinculación de Podemos con el 15M ha sido más simbólica que organizativa, ligando su narrativa de manera explícita con el movimiento del 15M (Lobera y Parejo, 2017). En suma, parte del éxito de un nuevo partido político dependería de aspectos organizativos pero, también, de que su identificación simbólica con un movimiento de protesta que contó con un alto nivel de apoyo.

Desde una perspectiva macro, Philippe Schmitter (2006) plantea una lista de doce factores que han podido favorecer la crisis de los partidos tradicionales y la aparición de nuevos partidos en Europa occidental desde los años setenta, y que pueden complementar la contextualización de nuestro caso de estudio. Entre estos, destacamos los siguientes: el impacto de crisis exógenas, el cambio de valores, la globalización-liberalización, la integración europea, el bajo crecimiento económico, el envejecimiento de la población, el papel de los medios de

${ }^{2}$ La estrategia de confluencia -Syriza significa "Coalición de la Izquierda"- está condicionada y favorecida por el 'premio' de 50 escaños que el sistema electoral griego otorga al partido más votado. Esta confluencia ha llevado a un nivel elevado de pluralismo interno, lo que, en ocasiones, ha conducido al faccionalismo a costa de la unidad (Tsakatika, 2015:95). 
comunicación, las innovaciones tecnológicas y la menor capacidad del Estado para satisfacer las demandas de la ciudadanía.

\section{METODOLOGÍA}

Nuestro estudio se centra en analizar cómo se transforma el apoyo a un movimiento de protesta (15M) en el voto a un nuevo partido político (Podemos) en los meses inmediatos a la aparición de este último. Nuestra hipótesis es que el apoyo al movimiento cristaliza electoralmente de manera heterogénea entre diferentes grupos sociales, según edad, nivel educativo y, principalmente, ideología política. Las nuevas evidencias que resulten de este análisis nos ayudarán a caracterizar la emergencia del nuevo partido.

Para ello, utilizamos datos de tres encuestas elaboradas por el Centro de Investigaciones Sociológicas (CIS), representativas de la población española de ambos sexos de 18 y más años. El Estudio 2905 entrevistó a 2.472 personas en junio de 2011, aproximadamente un mes después del inicio de las movilizaciones del 15M. El Estudio 3028, con una muestra de 2.464 personas, fue realizado entre mayo y julio de 2014 y recogió información detallada sobre los comicios al parlamento europeo celebrados en mayo de ese mismo año. Por último, el Estudio 3041 entrevistó a 2.480 personas en octubre de 2014 y contiene información sobre intención de voto a las diferentes opciones políticas de cara a unas eventuales elecciones generales.

En el Estudio 2905 de junio de 2011, se escogió como variable dependiente la simpatía hacia las protestas desarrolladas por el 15M. ¿Cuál es su opinión con relación a los acontecimientos protagonizados por este movimiento? Muy positiva, Más bien positiva, Ni positiva ni negativa (no leer), Más bien negativa, Muy negativa, N.S., N.C. En el Estudio de mayo-julio de 2014, se utilizó el recuerdo de voto a Podemos en las elecciones al PE del 25 de mayo: ¿Podría decirme el nombre del partido o coalición por el cual votó Ud. en las elecciones al Parlamento Europeo del pasado domingo 25 de mayo? En el Estudio 3041 de octubre de 2014 se escogió la intención de voto a Podemos: Suponiendo que mañana se celebrasen elecciones generales, es decir, al Parlamento español, ¿a qué partido votaría $U d$.? La utilización de esta última encuesta tiene como objetivo dar mayor fiabilidad y solidez a los resultados, ya que aporta una mayor base muestral en un momento (octubre de 2014) en el que la intención directa de voto a Podemos alcanzó el 17,4\%, por encima del PSOE (14,3\%) y el PP (11,7\%). Las variables independientes analizadas fueron el sexo, la edad, la situación ocupacional, el nivel educativo, el tamaño del municipio de residencia, la autoubicación ideológica y el recuerdo de voto en las elecciones generales de 2011.

Nuestra estrategia de investigación consistió, en primer lugar, en un análisis descriptivo que relaciona las tres variables dependientes con las independientes a través de tablas de contingencia, cuya significatividad estadística se mide a través del test de Chi-cuadrado. En segundo lugar, se realizaron tres modelos de regresión logística bivariada utilizando como variables dependientes la sim- 
patía hacia el $15 \mathrm{M}$-en la que se considera que el entrevistado tiene simpatía si manifiesta una visión 'muy positiva o más bien positiva' hacia las acciones del movimiento-, el recuerdo de voto a Podemos en las elecciones al PE de 2014 y la intención de voto a Podemos en octubre de 2014. El objetivo con este análisis fue conocer hasta qué punto operan las mismas variables en la simpatía hacia el $15 \mathrm{M}$ y en el apoyo electoral a Podemos.

En un tercer momento, y con el objetivo de superar la ausencia de encuestas que contengan, al mismo tiempo, la simpatía hacia el $15 \mathrm{M}$ y el apoyo a Podemos, proponemos el cálculo de un indicio de cristalización electoral (Ic). Esta magnitud se refiere a la transformación de la simpatía del movimiento social entre la opinión publica en apoyo electoral al nuevo partido político vinculado con ese movimiento. Cada nuevo partido político surgido de un mismo movimiento presenta un $I c$ diferente -así, por ejemplo, el Ic de Podemos será mayor que el Ic' de la Red Ciudadana Partido X. Al mismo tiempo, podemos calcular un $I c_{i}$ para cada segmento social, en función de variables clave. Esto nos permite constatar entre qué sectores sociales se produce una mayor o menor traducción de apoyo en votos, por lo que nos da una perspectiva caracterizadora de la formación del nuevo partido. Calculamos el índice de cristalización electoral para cada grupo de población $(i)$ de la siguiente manera:

Indice de cristalización electoral $l_{i}\left(I_{i}\right)=\frac{\text { Recuerdo de voto al partido político }_{i}}{\text { Simpatía hacia el movimiento } \text { social }_{i}} * 100$

Donde $i$ son los distintos grupos de población, segmentados por la variable $n$. Con el fin de medir la capacidad explicativa de cada una de las variables se utiliza un segundo indicador creado ad hoc para este trabajo: la ratio de variación, que indica, para cada variable independiente, la desviación entre la categoría (grupo social) que más convierte la simpatía por el $15 \mathrm{M}$ en voto a Podemos y la que menos. Informa, así, de la capacidad explicativa de dicha variable. Calculamos la ratio de variación para cada variable $n$ de la siguiente manera:

$$
\text { Ratio de variación } n_{n}=\frac{\max \left(I c_{n}\right)}{\min \left(I c_{n}\right)}
$$

El cálculo del índice de cristalización (Ic) y la ratio de variación nos ofrecen información acerca de cómo se ha consolidado el voto a Podemos entre los simpatizantes del 15M, evidenciando las diferencias entre distintos grupos sociales e identificando las variables con mayor dispersión.

Las premisas de nuestro trabajo son dos. En primer lugar, asumimos que existe una vinculación entre la base social del $15 \mathrm{M}$ y de Podemos, como se ha señalado en diversos estudios (véase, entre otros, Andretta y Della Porta, 2015; Martín, 2015; Tormeya y Feenstra, 2015; Calvo y Álvarez, 2015; Subirats, 2015; Toret, 2015). En concreto, creemos razonable asumir que el voto que obtiene Podemos en sus primeras elecciones (7,9\% del total) está contenido en el 74\% 
de simpatía del movimiento de protesta (Tabla 1), o al menos en una proporción suficiente para que el cálculo tenga sentido. En segundo lugar, creemos que existe una limitación temporal en la aplicación del índice a la primera fase de desarrollo de las nuevas alternativas electorales. En las elecciones municipales y autonómicas de 2015, donde la emergencia de plataformas municipalistas y confluencias estaría ligada igualmente al apoyo al 15M (Romanos y Sádaba, 2016; Calle, 2015; Subirats, 2015; Martín 2015), consideramos que no podemos aplicar nuestra metodología con garantías, ya que los cambios en la composición del electorado durante los primeros meses de 2015 -momento en el que tuvo lugar una dura confrontación en el campo de la comunicación electoral- sumado a la distorsión del recuerdo de apoyo al movimiento social (tendente a minimizarlo) pueden disminuir la fiabilidad del cálculo.

\section{RESULTADOS}

La tabla 1 muestra, en su primera columna, los porcentajes de personas que mostraban simpatía (opinión muy positiva o más bien positiva) hacia el $15 \mathrm{M}$ en junio de 2011, según diferentes variables. De acuerdo con la edad, las personas mayores de 64 años mostraban una simpatía marcadamente más reducida que el resto $(57,6 \%$, frente a un mínimo de 75,6\% entre quienes tenían entre 50 y 64 años). Los más jóvenes son quienes en mayor medida $(77,3 \%)$ mostraban simpatía por las manifestaciones del movimiento $15 \mathrm{M}$. Según recuerdo de voto en las últimas elecciones generales (2008), el 87,5\% de quienes afirmaban haber votado a IU (izquierda) mostraba simpatía hacia el $15 \mathrm{M}$, un porcentaje similar a quienes afirmaban haber votado al PSOE (centro-izquierda) $(83,7 \%)$ y a opciones más minoritarias $(85,3 \%)$. Entre quienes votaron en blanco o no votaron la simpatía se sitúa entre el 74,2\% y el 76,9\%. El apoyo es menor, aunque mayoritario, entre quienes votaron al PP (derecha), con el 59,1\% de simpatía hacia el $15 \mathrm{M}$ y a CIU (derecha nacionalista catalana), con el 53,3\%.

Según condición socioeconómica, se observa una simpatía muy significativa entre estudiantes $(83,3 \%)$, pequeños empresarios $(82,9 \%)$, técnicos o cuadros medios $(79,8 \%)$ y parados $(79,3 \%)$. La menor simpatía se encuentra entre las amas de casa $(61,7 \%)$, jubilados y pensionistas $(62,7 \%)$ y directores y profesionales $(67,9 \%)$. No parecen existir diferencias según estatus socioeconómico, aunque llama la atención que el mayor nivel de simpatía se observa en las clases altas/medias altas (79\%). Según nivel de estudios, el 78,4\% de quienes tienen estudios universitarios simpatiza con el $15 \mathrm{M}$, en contraste con el $69,5 \%$ de quienes tienen como máximo estudios primarios. La simpatía se incrementa visiblemente a medida que el entrevistado se sitúa en posiciones ideológicas más hacia la izquierda, con un máximo en la extrema izquierda $(91,2 \%)$ y un mínimo en la extrema derecha (44\%). No se aprecian diferencias significativas entre hombres y mujeres, ni según el tamaño del hábitat.

La segunda columna de la tabla 1 muestra el recuerdo de voto a Podemos en las elecciones al PE de mayo de 2014. El 8,9\% afirmó haber votado a Podemos 
en las citadas elecciones. Según edad, solo lo hizo el 3,1\% de los mayores de 64 años, por el 11,8\% de quienes tenían entre 18 y 34 años. El 22,4\% de quienes votaron a IU en las elecciones generales de 2011 afirman haber votado a Podemos en 2014, el 11,7\% entre quienes votaron al PSOE, y el 10,6\% y 9,8\% entre quienes no tenían derecho a voto o votaron en blanco, respectivamente. Solo el 3,3\% de quienes votaron al PP en 2011 afirmaron haber optado por Podemos en 2014. Según condición socioeconómica, el mayor recuerdo de voto a Podemos se observa entre los estudiantes $(15,5 \%)$, los obreros $(13 \%)$ los técnicos y cuadros medios $(11,4 \%)$, y los empleados de oficinas y servicios $(11,1 \%)$. Jubilados, pensionistas y trabajadoras no remuneradas $(4,6 \%)$ y los directores y profesionales $(5,3 \%)$ son quienes presentan un menor recuerdo de voto a esta formación. De acuerdo con el nivel de estudios, se observa una gran diferencia entre quienes no superaron los estudios primarios $(3,5 \%)$ y el resto (a partir del $10,3 \%)$. Podemos recogió votos fundamentalmente de quienes se autoubican en la izquierda, mientras que su presencia entre la derecha es casi inexistente. Su apoyo también fue mayor en ciudades grandes, con más de un $11 \%$ de recuerdo de voto en municipios de más de 100.000 habitantes, en contraste con el 6\% de los municipios de 10.000 habitantes o menos.

La tercera columna de la tabla 1 muestra la intención de voto a Podemos tan solo cinco meses tras las elecciones al PE, en octubre de 2014. La intención de voto en ese momento es muy superior al recuerdo de voto de junio del mismo año. Se aprecia una intención de voto superior entre los hombres $(24,6 \%$ frente a 20,4\% de las mujeres) y entre los menores de 65 años, especialmente entre los menores de 34 años (28,9\%, frente al 7,3\% de los mayores de 64 años). De manera similar a los datos junio de 2014, la intención de voto es muy elevada entre los exvotantes de IU (47\%) y PSOE $(29,2 \%)$, y se incrementa significativamente entre los exvotantes de UPYD $(35,7 \%)$. La mayor intención de voto se da entre los estudiantes $(29,9 \%)$, los obreros $(28,3 \%)$ y los técnicos y cuadros medios $(27,9 \%)$, y la menor entre los jubilados y pensionistas $(11,1 \%)$ y los trabajadores domésticos no remunerados $(13,2 \%)$. Entre quienes alcanzan como máximo estudios primarios, la previsión de votar a Podemos es mucho menor que quienes tienen mayor nivel de estudios $(8,8 \%$, por el $27,3 \%$ y el $25,8 \%$ de quienes tienen estudios secundarios o universitarios, respectivamente). De manera similar a lo encontrado en junio de 2014, existe una mayor intención de voto entre quienes se autoubican en la izquierda y entre quienes viven en municipios más poblados. 
Tabla 1. Simpatía hacia el 15M en junio 2011, recuerdo de voto a Podemos en junio 2014 e intención de voto a Podemos en octubre de 2014, según diferentes variables

\begin{tabular}{|c|c|c|c|c|c|c|c|c|c|c|}
\hline & & \multicolumn{3}{|c|}{$\begin{array}{l}\text { Simpatía hacia el } \\
\text { 15M Junio } 2011\end{array}$} & \multicolumn{3}{|c|}{$\begin{array}{c}\text { Recuerdo de voto } \\
\text { a Podemos Junio } \\
\mathbf{2 0 1 4} \\
\end{array}$} & \multicolumn{3}{|c|}{\begin{tabular}{|c} 
Intención de voto \\
a Podemos Oct. \\
2014 \\
\end{tabular}} \\
\hline & & $\mathrm{N}$ & $\%$ & $\begin{array}{l}\text { Sig. Chi- } \\
\text { cuadrado }\end{array}$ & $\mathrm{N}$ & $\%$ & $\begin{array}{l}\text { Sig. Chi- } \\
\text { cuadrado }\end{array}$ & $\mathrm{N}$ & $\%$ & $\begin{array}{l}\text { Sig. Chi- } \\
\text { cuadrado }\end{array}$ \\
\hline \multirow{2}{*}{ Sexo } & Hombre & 661 & 73,2 & \multirow{2}{*}{0,627} & 105 & 9,5 & \multirow{2}{*}{0,279} & 243 & 24,6 & \multirow{2}{*}{0,026} \\
\hline & Mujer & 676 & 74,2 & & 92 & 8,2 & & 194 & 20,4 & \\
\hline \multirow{4}{*}{ Edad } & $18-34$ & 449 & 77,3 & \multirow{4}{*}{0,000} & 70 & 11,8 & \multirow{4}{*}{0,000} & 153 & 28,9 & \multirow{4}{*}{0,000} \\
\hline & $35-49$ & 417 & 76,4 & & 66 & 9,8 & & 152 & 25,8 & \\
\hline & $50-64$ & 316 & 75,6 & & 47 & 9,4 & & 104 & 23,7 & \\
\hline & 65 y más & 155 & 57,6 & & 14 & 3,1 & & 28 & 7,3 & \\
\hline \multirow{10}{*}{$\begin{array}{l}\text { Recuerdo de } \\
\text { voto }\end{array}$} & & \multicolumn{3}{|c|}{ Generales 2008} & \multicolumn{3}{|c|}{ Generales 2011} & \multicolumn{3}{|c|}{ Generales 2011} \\
\hline & PSOE & 81 & 83,7 & & 64 & 11,7 & & 126 & 28,2 & \\
\hline & PP & 141 & 59,1 & & 17 & 3,3 & & 32 & 7,4 & \\
\hline & IU & 12 & 87,5 & & 39 & 22,4 & & 71 & 47,7 & \\
\hline & UPyD & & & & 5 & 7,8 & & 20 & 35,7 & \\
\hline & $\mathrm{CIU}$ & 21 & 53,3 & 0,000 & 1 & 1,6 & 0,000 & 4 & 8,3 & 0,000 \\
\hline & Otros partidos & 16 & 85,3 & & 13 & 8,3 & & 41 & 26,8 & \\
\hline & $\begin{array}{l}\text { Sin derecho al } \\
\text { voto }\end{array}$ & 15 & 76,9 & & 12 & 10,6 & & 11 & 18,6 & \\
\hline & Votó en blanco & 16 & 74,2 & & 6 & 9,8 & & 22 & 29,5 & \\
\hline & No votó & 70 & 75,7 & & 19 & 5,8 & & 73 & 18,9 & \\
\hline \multirow{9}{*}{$\begin{array}{l}\text { Condición } \\
\text { socio- } \\
\text { económica }\end{array}$} & $\begin{array}{l}\text { Directores y } \\
\text { profesionales }\end{array}$ & 55 & 67,9 & \multirow{9}{*}{0,000} & 5 & 5,3 & \multirow{9}{*}{0,000} & 19 & 25,3 & \multirow{9}{*}{0,000} \\
\hline & $\begin{array}{l}\text { Técnicos y } \\
\text { cuadros medios }\end{array}$ & 166 & 79,8 & & 28 & 11,4 & & 58 & 27,9 & \\
\hline & $\begin{array}{l}\text { Pequeños } \\
\text { empresarios }\end{array}$ & 63 & 82,9 & & 9 & 10,1 & & 15 & 23,1 & \\
\hline & $\begin{array}{l}\text { Empleados } \\
\text { de oficina y } \\
\text { servicios }\end{array}$ & 105 & 73,9 & & 40 & 11,1 & & 75 & 24,5 & \\
\hline & $\begin{array}{l}\text { Obreros } \\
\text { cualificados, no } \\
\text { cualificados y } \\
\text { agricultores }\end{array}$ & 246 & 76,6 & & 14 & 13,0 & & 30 & 28,3 & \\
\hline & $\begin{array}{l}\text { Jubilados y } \\
\text { pensionistas }\end{array}$ & 220 & 62,7 & & 23 & 4,6 & & 51 & 11,1 & \\
\hline & Parados & 317 & 79,3 & & 52 & 9,9 & & 140 & 29,9 & \\
\hline & $\begin{array}{l}\text { Estudiantes } \\
\text { Trabajo }\end{array}$ & 70 & 83,3 & & 17 & 15,5 & & 24 & 25,0 & \\
\hline & $\begin{array}{l}\text { doméstico no } \\
\text { rem }\end{array}$ & 74 & 61,7 & & 7 & 4,6 & & 17 & 13,2 & \\
\hline
\end{tabular}




\begin{tabular}{|c|c|c|c|c|c|c|c|c|c|c|}
\hline & \multicolumn{3}{|c|}{$\begin{array}{l}\text { Simpatía hacia el } \\
\text { 15M Junio } 2011\end{array}$} & \multicolumn{3}{|c|}{$\begin{array}{c}\text { Recuerdo de voto } \\
\text { a Podemos Junio } \\
2014\end{array}$} & \multicolumn{3}{|c|}{$\begin{array}{c}\text { Intención de voto } \\
\text { a Podemos Oct. } \\
2014\end{array}$} \\
\hline & & $\mathrm{N}$ & $\%$ & $\begin{array}{l}\text { Sig. Chi- } \\
\text { cuadrado }\end{array}$ & $\mathrm{N}$ & $\%$ & $\begin{array}{l}\text { Sig. Chi- } \\
\text { cuadrado }\end{array}$ & $\mathrm{N}$ & $\%$ & $\begin{array}{l}\text { Sig. Chi- } \\
\text { cuadrado }\end{array}$ \\
\hline \multirow{3}{*}{$\begin{array}{l}\text { Nivel de } \\
\text { estudios }\end{array}$} & $\begin{array}{l}\text { Primarios o } \\
\text { menos }\end{array}$ & 573 & 69,5 & \multirow{3}{*}{0,001} & 18 & 3,5 & \multirow{3}{*}{0,000} & 41 & 8,8 & \multirow{3}{*}{0,000} \\
\hline & $\begin{array}{l}\text { Secundaria o } \\
\text { FP }\end{array}$ & 431 & 76,3 & & 127 & 10,3 & & 304 & 27,3 & \\
\hline & Universitarios & 330 & 78,4 & & 51 & 10,9 & & 92 & 25,8 & \\
\hline \multirow{10}{*}{ Ideología } & $\begin{array}{l}\text { Extrema } \\
\text { izquierda }\end{array}$ & 62 & 91,2 & \multirow{10}{*}{0,000} & 17 & 17,5 & \multirow{10}{*}{0,000} & 52 & 44,8 & \multirow{10}{*}{0,000} \\
\hline & 2,00 & 72 & 77,4 & & 28 & 21,4 & & 59 & 42,4 & \\
\hline & 3,00 & 234 & 85,7 & & 63 & 17,1 & & 124 & 37,0 & \\
\hline & 4,00 & 214 & 85,9 & & 42 & 12,2 & & 80 & 28,1 & \\
\hline & 5,00 & 310 & 74,9 & & 25 & 6,1 & & 71 & 21,5 & \\
\hline & 6,00 & 118 & 65,9 & & 4 & 2,1 & & 11 & 7,3 & \\
\hline & 7,00 & 57 & 61,3 & & 1 & 0,7 & & 5 & 4,6 & \\
\hline & 8,00 & 41 & 50,6 & & 0 & 0,0 & & 3 & 3,4 & \\
\hline & 9,00 & 12 & 52,2 & & 0 & 0,0 & & 0 & 0,0 & \\
\hline & $\begin{array}{l}\text { Extrema } \\
\text { derecha }\end{array}$ & 11 & 44,0 & & 1 & 3,6 & & 0 & 0,0 & \\
\hline \multirow{4}{*}{$\begin{array}{l}\text { Tamaño de } \\
\text { hábitat }\end{array}$} & $0-10.000$ & 272 & 74,3 & \multirow{4}{*}{0,983} & 29 & 6,0 & \multirow{4}{*}{0,007} & 75 & 17,9 & \multirow{4}{*}{0,002} \\
\hline & $10.001-100.000$ & 486 & 73,4 & & 67 & 7,9 & & 150 & 20,6 & \\
\hline & $\begin{array}{l}100.001- \\
1.000 .000\end{array}$ & 417 & 73,4 & & 76 & 11,4 & & 156 & 26,5 & \\
\hline & $\begin{array}{l}\text { Más de } \\
1.000 .000\end{array}$ & 162 & 74,3 & & 25 & 11,0 & & 56 & 27,7 & \\
\hline
\end{tabular}

Elaboración propia a partir de los estudios CIS 2905 junio 2011; 3028 mayo-junio 2014; 3041 octubre 2014.

La tabla 2 muestra los análisis de regresión logística. El primero toma como variable dependiente la simpatía hacia el $15 \mathrm{M}$. La única variable del modelo en la que se observan diferencias significativas $(\mathrm{p}<0,05)$ es el recuerdo de voto en las últimas elecciones generales. En comparación con los votantes del PSOE en 2008, la simpatía al $15 \mathrm{M}$ es significativamente menor entre los votantes de $\mathrm{PP}(\mathrm{OR}=0,296)$, $\mathrm{CIU}(\mathrm{OR}=0,223)$, entre quienes no votaron $(\mathrm{OR}=0,548)$, votaron en blanco $(\mathrm{OR}=0,459)$ y entre quienes no tenían derecho a voto en 2008 $(\mathrm{OR}=0,451)$.

El segundo análisis de regresión logística (tabla 2) toma como variable dependiente el recuerdo de voto a Podemos en 2014. En comparación con quienes votaron al PSOE en las elecciones generales de 2011, el recuerdo de voto a Podemos es significativamente más reducido entre quienes votaron al PP $(\mathrm{OR}=0,265)$, a $\mathrm{CIU}(\mathrm{OR}=0,117)$ o no votaron $(\mathrm{OR}=0,396)$, y mayor entre quienes votaron a IU $(\mathrm{OR}=1,786)$. En relación con quienes tienen estudios primarios o menos, quienes tienen estudios secundarios $(\mathrm{OR}=2,129)$ o universitarios $(\mathrm{OR}=2,459)$ tienen un recuerdo de voto mayor; lo mismo ocurre entre quienes 
viven en ciudades con entre 100.001 y 1.000 .000 de habitantes $(\mathrm{OR}=1,744)$, respecto a quienes viven en municipios con menos de 10.001 habitantes.

Tabla 2. Modelos de regresión logística de simpatía hacia el 15M en 2011, recuerdo de voto a Podemos en junio de 2014 e intención de voto a Podemos en

\begin{tabular}{|c|c|c|c|c|c|c|}
\hline & \multicolumn{2}{|c|}{$\begin{array}{l}\text { Simpatía hacia } \\
15 \mathrm{M} \text { junio } 2011\end{array}$} & \multicolumn{2}{|c|}{$\begin{array}{c}\text { Recuerdo de } \\
\text { voto a Podemos } \\
\text { junio } 2014 \\
\end{array}$} & \multicolumn{2}{|c|}{$\begin{array}{c}\text { Intención } \\
\text { de voto a } \\
\text { Podemos } \\
\text { octubre 2014 } \\
\end{array}$} \\
\hline & Sig. & $\operatorname{Exp}(B)$ & Sig. & $\operatorname{Exp}(B)$ & Sig. & $\operatorname{Exp}(B)$ \\
\hline $18-34$ & 0,180 & & 0,262 & & 0,043 & \\
\hline $35-49$ & 0,954 & 1,010 & 0,720 & 0,925 & 0,075 & 0,752 \\
\hline $50-64$ & 0,322 & 1,224 & 0,865 & 0,959 & 0,762 & 0,945 \\
\hline 65 y más & 0,280 & 0,721 & 0,062 & 0,396 & 0,036 & 0,485 \\
\hline PSOE & 0,000 & & 0,000 & & 0,000 & \\
\hline PP & 0,000 & 0,296 & 0,000 & 0,265 & 0,000 & 0,200 \\
\hline IU & 0,485 & 1,265 & 0,014 & 1,786 & 0,009 & 1,725 \\
\hline UPyD & & & 0,142 & 0,484 & 0,832 & 0,934 \\
\hline CIU & 0,000 & 0,223 & 0,036 & 0,117 & 0,007 & 0,234 \\
\hline Otros partidos & 0,979 & 1,008 & 0,054 & 0,531 & 0,201 & 0,753 \\
\hline Sin derecho al voto & 0,038 & 0,451 & 0,132 & 0,471 & 0,023 & 0,382 \\
\hline Votó en blanco & 0,016 & 0,459 & 0,068 & 0,494 & 0,622 & 0,870 \\
\hline No voto & 0,002 & 0,548 & 0,001 & 0,396 & 0,023 & 0,382 \\
\hline Primario & 0,214 & & 0,052 & & 0,000 & \\
\hline Secundaria o FP & 0,083 & 1,320 & 0,026 & 2,129 & 0,000 & 2,504 \\
\hline Universita & 0,333 & 1,212 & 0,017 & 2,459 & 0,003 & 2,143 \\
\hline Jubilad & 0,207 & & 0,282 & & 0,674 & \\
\hline profesionales & 0,937 & 1,029 & 0,251 & 0,512 & 0,420 & 1,349 \\
\hline $\begin{array}{l}\text { Técnicos y cuadros } \\
\text { medios }\end{array}$ & 0,229 & 1,478 & 0,497 & 0,753 & 0,529 & 1,214 \\
\hline Pequeños empresarios & 0,108 & 1,926 & 0,752 & 1,169 & 0,533 & 1,287 \\
\hline $\begin{array}{l}\text { Empleados de oficina y } \\
\text { servicios }\end{array}$ & 0,801 & 1,087 & 0,731 & 0,876 & 0,630 & 1,149 \\
\hline $\begin{array}{l}\text { Obreros cualificados, no } \\
\text { cualificados y agricultores }\end{array}$ & 0,260 & 1,372 & 0,492 & 1,374 & 0,344 & 1,390 \\
\hline Parados & 0,065 & 1,663 & 0,989 & 0,995 & 0,113 & 1,538 \\
\hline Estudiant & 0,109 & 2,055 & 0,269 & 1,716 & 0,828 & 1,094 \\
\hline $\begin{array}{l}\text { Trabajo domestico no } \\
\text { remun. }\end{array}$ & 0,423 & 0,791 & 0,145 & 0,422 & 0,791 & 0,906 \\
\hline Mujer (hombre) & 0,530 & 1,088 & 0,442 & 0,876 & 0,109 & 0,816 \\
\hline $0-10.000$ & 0,903 & & 0,080 & & 0,020 & \\
\hline $10.001-100.000$ & 0,462 & 0,877 & 0,532 & 1,170 & 0,543 & 1,113 \\
\hline $100.001-1.000 .000$ & 0,599 & 0,907 & 0,027 & 1,744 & 0,017 & 1,535 \\
\hline Más de 1.000 .000 & 0,807 & 0,943 & 0,184 & 1,550 & 0,024 & 1,686 \\
\hline Constante & 0,000 & 3,828 & 0,000 & 0,075 & 0,000 & 0,188 \\
\hline
\end{tabular}




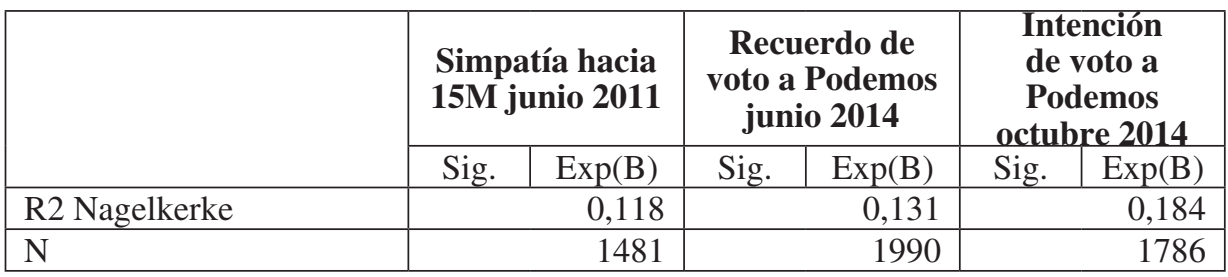

Elaboración propia a partir de los estudios del CIS 2905 Junio 2011, 3028 mayo-junio 2014 y 3041 octubre 2014.

La tabla 3 muestra, para junio y octubre de 2014, el índice de cristalización electoral según diferentes variables, es decir, las probabilidades de que ese grupo social convierta su simpatía por el 15M en voto a Podemos. En junio se observan bajos niveles de cristalización (Ic) entre los que viven en municipios con menos de 10.000 habitantes $(8,1)$, entre quienes tienen un nivel educativo bajo $(5,1)$, entre los jubilados y pensionistas $(7,4)$ y las amas de casa $(7,5)$, así como entre las personas que se ubican ideológicamente a la derecha o votan al PP $(5,6)$. Asimismo, se observan índices de cristalización significativamente más bajos entre los mayores de 64 años $(5,4)$. Por otro lado, la simpatía por el movimiento de protesta parece cristalizar más fácilmente en voto a Podemos entre los votantes de IU $(25,6)$ que entre los del PSOE $(14,0)$-aunque estos, por ser un colectivo mayor, supongan una mayor proporción del conjunto de votantes de Podemos-. Por su parte, las variables que presentan mayores diferencias de cristalización entre sus segmentos (ratio de variación) son el recuerdo de voto en las elecciones generales de 2011, con una ratio de 8,33 entre el máximo (votantes de IU) y el mínimo (votantes del PP) ${ }^{3}$, seguida por la edad $(2,81)$ y el nivel de estudios $(2,73)$.

${ }^{3}$ Para este cálculo hemos utilizado únicamente los datos correspondientes a partidos con presencia en el conjunto de las Comunidades Autónomas 
Tabla 3. Índices de cristalización del voto en junio y octubre de 2014, según diferentes variables

\begin{tabular}{|c|c|c|c|c|c|}
\hline \multirow{2}{*}{\multicolumn{2}{|c|}{ Q }} & \multicolumn{2}{|c|}{$\begin{array}{l}\text { Recuerdo de voto a } \\
\text { Podemos junio } 2014\end{array}$} & \multicolumn{2}{|c|}{$\begin{array}{c}\text { Intención de voto a } \\
\text { Podemos octubre } 2014\end{array}$} \\
\hline & & $\begin{array}{c}\text { Índice de } \\
\text { cristalización } \\
\text { (Ic) }\end{array}$ & $\begin{array}{c}\text { Ratio de } \\
\text { variación } \\
\text { (máx/mín) }\end{array}$ & $\begin{array}{l}\text { Índice de } \\
\text { cristalización } \\
\text { (Ic) }\end{array}$ & $\begin{array}{l}\text { Ratio de } \\
\text { variación } \\
\text { (máx/mín) }\end{array}$ \\
\hline \multirow{2}{*}{ Sexo } & Hombre & 13,0 & \multirow[t]{2}{*}{1,17} & 33,6 & \multirow[t]{2}{*}{1,22} \\
\hline & Mujer & 11,1 & & 27,5 & \\
\hline \multirow{4}{*}{ Edad } & $18-34$ & 15,2 & \multirow[t]{4}{*}{2,81} & 37,4 & \multirow[t]{4}{*}{2,93} \\
\hline & $35-49$ & 12,8 & & 33,7 & \\
\hline & $50-64$ & 12,4 & & 31,4 & \\
\hline & 65 y más & 5,4 & & 12,8 & \\
\hline \multirow{9}{*}{$\begin{array}{l}\text { Recuerdo } \\
\text { de voto en } \\
\text { elecciones } \\
\text { generales } \\
2011\end{array}$} & PSOE & 14,0 & \multirow[t]{9}{*}{8,33} & 33,7 & \multirow[t]{9}{*}{4,37} \\
\hline & PP & 5,5 & & 12,5 & \\
\hline & IU & 25,6 & & 54,5 & \\
\hline & UPyD & ND & & ND & \\
\hline & CIU & 3,1 & & 15,6 & \\
\hline & Otros partidos & 9,7 & & 31,4 & \\
\hline & $\begin{array}{l}\text { Sin derecho al } \\
\text { voto }\end{array}$ & 13,8 & & 24,2 & \\
\hline & Votó en blanco & 13,3 & & 39,7 & \\
\hline & No votó & 7,6 & & 24,9 & \\
\hline \multirow{11}{*}{$\begin{array}{l}\text { Condición } \\
\text { socio- } \\
\text { económica }\end{array}$} & $\begin{array}{l}\text { Directores y } \\
\text { profesionales }\end{array}$ & 7,8 & \multirow[t]{11}{*}{2,51} & 37,3 & \multirow[t]{11}{*}{2,13} \\
\hline & $\begin{array}{l}\text { Técnicos y } \\
\text { cuadros medios }\end{array}$ & 14,3 & & 34,9 & \\
\hline & $\begin{array}{l}\text { Pequeños } \\
\text { empresarios }\end{array}$ & 12,2 & & 27,8 & \\
\hline & $\begin{array}{l}\text { Empleados } \\
\text { de oficina y }\end{array}$ & 15,0 & & 33,1 & \\
\hline & \begin{tabular}{|l} 
servicios \\
Obreros
\end{tabular} & & & & \\
\hline & $\begin{array}{l}\text { cualificados, no } \\
\text { cualificados } \mathrm{y}\end{array}$ & 16,9 & & 36,9 & \\
\hline & $\begin{array}{l}\text { cuanincados y } \\
\text { agricultores }\end{array}$ & & & & \\
\hline & $\begin{array}{l}\text { Jübilados y } \\
\text { pensionistas }\end{array}$ & 7,4 & & 17,7 & \\
\hline & Parados & 12,5 & & 37,7 & \\
\hline & $\begin{array}{l}\text { Estudiantes } \\
\text { Trabajo }\end{array}$ & 18,5 & & 30,0 & \\
\hline & $\begin{array}{l}\text { doméstico no } \\
\text { remun. }\end{array}$ & 7,5 & & 21,4 & \\
\hline \multirow{3}{*}{$\begin{array}{l}\text { Nivel de } \\
\text { estudios }\end{array}$} & $\begin{array}{l}\text { Primarios o } \\
\text { menos }\end{array}$ & 5,1 & \multirow[t]{3}{*}{2,73} & 12,7 & \multirow[t]{3}{*}{2,82} \\
\hline & Secundaria o & 13,4 & & 35,8 & \\
\hline & Universitarios & 13,9 & & 32,9 & \\
\hline
\end{tabular}




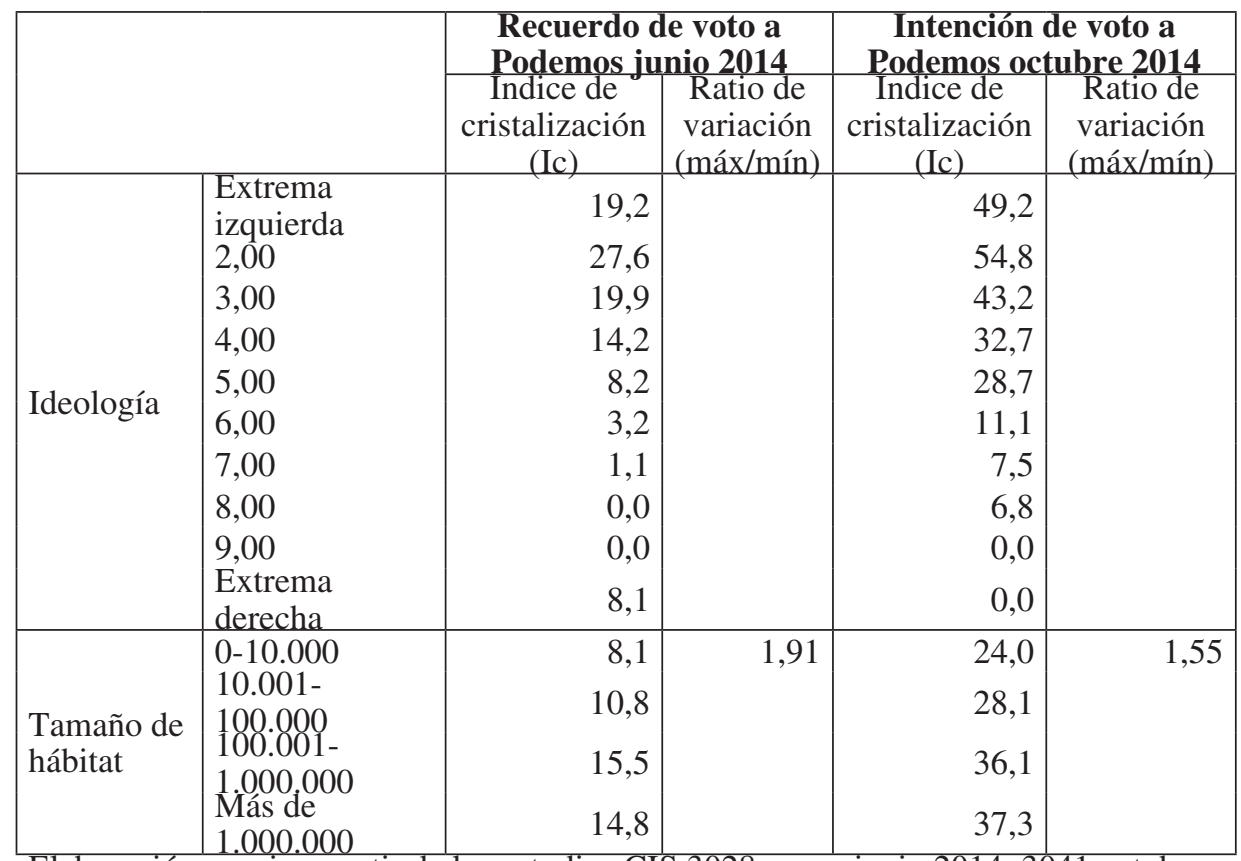

Elaboración propia a partir de los estudios CIS 3028 mayo-junio 2014; 3041 octubre 2014.

En términos generales, en octubre de 2014 los $I c$ son mucho mayores que en junio, dado que la intención de voto a Podemos se había incrementado significativamente (tabla 3). De manera similar a lo encontrado cuatro meses antes, se observan mayores niveles de cristalización entre los exvotantes de IU $(54,5)$, PSOE $(33,7)$ y quienes votaron en blanco en las últimas elecciones generales $(39,7)$, entre quienes tenían mayores niveles de estudios (por encima de 35,8) y entre quienes vivían en municipios más grandes (a partir de 36,1 entre quienes viven en municipios de más de 100.000 habitantes). Resulta llamativo el aumento en el $I c$ entre los directivos y profesionales (37,3). Asimismo, se mantiene un Ic muy reducido entre los mayores de 64 años, los jubilados y pensionistas y entre los trabajadores domésticos no remunerados. La mayor variabilidad entre grupos dentro de las variables analizadas (Ratio de variación) se observa, al igual que en junio, en la ideología, el recuerdo de voto (aunque se reduce significativamente y pasa de 8,33 a 4,37$)$, la edad $(2,93)$ y el nivel de estudios $(2,82)$. 


\section{DISCUSIÓN}

El análisis constata que el apoyo a las movilizaciones del 15M fue 'transversal' entre los distintos sectores sociales, ya que fue mayoritario entre los distintos grupos de edad, sexo, hábitat, ideología, nivel educativo, nivel económico y situación ocupacional, si bien se produjo con intensidades distintas en algunos de ellos. ${ }^{4}$ Esta transversalidad es similar a la observada en la erosión del apoyo político y la desafección política durante las últimas décadas en España (Lobera y Ferrándiz, 2013; Muro y Vidal, 2016).

En segundo lugar, los resultados muestran que los factores que condicionan la simpatía por el movimiento son distintos a los que condicionan el voto. No todos los grupos de población cristalizan de igual forma su simpatía por el 15M. Mientras que el apoyo a las protestas tenía un marcado carácter transversal, en el voto a Podemos se intensifica sensiblemente el factor ideológico. Estos resultados pueden resultar llamativos, teniendo en cuenta que Podemos trató reiteradamente de desmarcarse del eje izquierda-derecha durante sus primeros dos años de existencia y que, posteriormente, se explicitó una dura competencia interna en torno al debate entre transversalidad y polarización (Lobera y Parejo, 2017). De alguna manera, la búsqueda de la transversalidad de la primera fase de Podemos contrasta con su primer electorado, más ideologizado. El dilema posterior entre politización y transversalidad aludiría, según los datos, a la disyuntiva de orientar la estrategia de Podemos hacia afianzar el voto duro (los votantes ideologizados) o hacia un eventual crecimiento entre votantes menos ideologizados.

Así, el análisis de los índices de cristalización electoral muestra que la simpatía por el movimiento cristaliza más fácilmente en voto a Podemos en mayo de 2014 entre los votantes de IU (izquierda) que entre los del PSOE (centro-izquierda) -aunque estos últimos, por ser un colectivo mayor, supongan una mayor proporción del conjunto de votantes de Podemos-. La menor cristalización en voto se observa entre quienes votaron al Partido Popular. Es decir, buena parte de los votantes del Partido Popular (59\%) simpatizaban con las movilizaciones del 15M, -e incluso expresaban su apoyo a sus reivindicaciones- aunque la proporción de quienes transformaron ese apoyo en voto a Podemos fue sensiblemente más baja que entre los otros partidos. En el caso de los votantes de la ya desaparecida CiU, a su ubicación en el centroderecha se añade la dimensión nacionalista -alejada del planteamiento de Podemos- por lo que no extraña observar un bajo índice de cristalización electoral entre ellos. Finalmente, también se registra un $I c$ reducida entre quienes votan a "Otros partidos". Esto último apunta a que los votantes de partidos minoritarios, a pesar de expresar una elevada simpatía por el movimiento de protesta -y posiblemente también por el nuevo partido-, seguían estando satisfechos con sus opciones electorales minoritarias y no buscaron una nueva opción electoral para canalizar su voto en mayo de 2014. Es decir, el voto a Podemos en 2014 aparece vinculado fundamentalmente con

4 Este análisis coincide con el realizado con otras bases de datos en estudios precedentes (Lobera y Sampedro, 2014; Sampedro y Lobera, 2014).

EMPIRIA. Revista de Metodología de Ciencias Sociales. N. 38 septiembre-diciembre, 2017, pp. 151-176. ISSN: 1139-5737, DOI/empiria.38.2018.19715 
la falta de credibilidad de los partidos tradicionales entre sus propios votantes. Este fenómeno se corresponde con lo observado en el caso de Syriza en Grecia por Verney (2014).

Por otro lado, las personas más involucradas en el activismo del $15 \mathrm{M}$ tenían un perfil ideológico marcado. Portos y Masullo (2017) muestran que los factores de movilización en el 15M fueron principalmente la ideología (autoubicación ideológica) y el activismo previo. Este perfil activista coincide con el votante de Podemos en las elecciones al PE, como señala el análisis de Fernández-Albertos (2015). Además, la simpatía por el 15M es más extensa entre los sectores de la izquierda y son estos los que votan en mayor proporción por la nueva formación política. Los factores que impulsan el activismo en el 15M, pues, son similares a los que impulsan el voto a Podemos en las elecciones al PE de 2014, apuntando a la existencia de una continuidad entre la movilización del $15 \mathrm{M}$ y el voto a Podemos.

Este perfil se transformará en su fase de crecimiento, como se aprecia en las tablas de resultados 1,2 y 3 . Sin embargo, en la primera convocatoria electoral a la que se presenta Podemos - unas elecciones, además, de segundo orden-, el voto puede considerarse como una forma más de movilización. En esta primera fase, el voto se convierte en una extensión de las protestas en el ámbito electoral, movilizando a aquellos colectivos que habían estado más implicados en ellas. Así, los resultados apuntan, en un sentido simétricamente inverso al planteado por Kasse (2007), que cabe entender el voto a un nuevo partido vinculado a la protesta como una forma institucionalizada de extender el posicionamiento político crítico desarrollado más allá del ciclo de protesta. El caso que estudiamos, lejos de ser una excepción, se inscribiría dentro de un marco común de extensión de los movimientos. Como señalan McAdam y Tarrow (2011: 165), frecuentemente los movimientos sociales incluyen el uso de la "opción electoral" entre sus tácticas y estrategias. Su excepcionalidad estaría asociada con los tiempos y magnitudes con la que se produce esta extensión electoral.

Es la continuidad con la protesta lo que caracteriza la fase emergencia de Podemos, tanto en la base social de su primer electorado, como hemos visto, como en lo que se refiere a su aspecto narrativo-simbólico. Su relación con la protesta es, pues, central para la caracterización del nuevo partido. En este sentido, el voto a Podemos no sería tanto un voto protesta como un voto de la protesta. Van der Eijk et al. (1996) definen al "votante protesta" como aquel que no vota por un partido ideológicamente cercano ni realiza un voto estratégico. El perfil de votante de Podemos, en cambio, sí presenta un voto ideológico -vinculado a la narrativa del 15M-. Por otro lado, los propios líderes de Podemos han subrayado y explotado electoralmente esta continuidad entre la protesta y la movilización electoral.

La distinta capitalización que ha obtenido Podemos de las simpatías por este movimiento entre diferentes sectores de la población está en la base explicativa tanto de su éxito como de los límites en su crecimiento. En línea con el marco comunicativo del 15M, el nuevo partido insistió, durante sus dos primeros años de existencia, en desmarcarse del eje izquierda-derecha y en establecer el nuevo 
marco de nueva y vieja política el eje de renovación de la política, de regeneración democrática ${ }^{5}$. A pesar de esos esfuerzos, los datos apuntan a que la emergencia electoral de Podemos estuvo fuertemente marcada por las preferencias políticas previas y por los sectores sociales vinculados con una participación más activa en las movilizaciones. Asimismo, tras sus buenos resultados en las elecciones al EP, Podemos se nutrió orgánicamente con parte del activismo inicial del 15M -en especial de los activistas jóvenes de "Juventud sin Futuro" y la lucha contra la reforma universitaria-.

La vinculación narrativo-simbólica y de base social con las protestas de 2011 ayuda a explicar el rápido crecimiento que experimentó el nuevo partido político, aunque estas son dos condiciones necesarias pero no suficientes explicar la emergencia de Podemos, ya que otras formaciones más cercanas de la lógica organizativa y los contenidos del núcleo movilizador del $15 \mathrm{M}$-como la Red Ciudadana Partido X- no lograron obtener un apoyo electoral comparable. Así, la explicación de la emergencia de Podemos debe complementarse con otros factores sobre los que no profundizaremos aquí pero que se han apuntado anteriormente (Cordero y Montero, 2015; Fernández-Albertos, 2015; Lobera, 2015): el contexto electoral de segundo orden presente en las elecciones de 2014, la visibilidad mediática, el uso del líder político en la fase de emergencia del partido, la habilidad en el debate público, la novedad ("sin facturas pendientes"), el enfoque transversal del discurso político y la debilidad de otras formaciones existentes para capitalizar el creciente volumen de votantes huérfanos.

Por otro lado, los resultados muestran un Ic significativamente más bajo entre los mayores de 64 años. Esto es consistente con la hipótesis de que, tanto las movilizaciones como el partido, se articulan en torno a la ruptura del consenso de la Transición, interpelando especialmente a las generaciones que no votaron el referéndum de la Constitución en 1978, es decir, entre los menores de 54 años (Martínez, 2012; Fernández-Savater, 2012; Moreno-Caballud, 2013). Adicionalmente, los datos muestran más intensamente entre los jóvenes la desafección hacia los partidos tradicionales y el voto por la nueva formación política. Estas observaciones son compatibles con distintas teorías previas que pueden ser complementarias, vinculando la insatisfacción política de los jóvenes con la crisis económica. Schmitter (2006) observó que el desempleo empuja a los jóvenes hacia los nuevos partidos en otras democracias europeas, convergiendo aquí la situación más elevada del desempleo juvenil que en cualquier otro país europeo -con la excepción de Grecia, en la que Syriza alcanzó el poder-.

En una línea similar, los resultados también son compatibles con las hipótesis planteadas por Francés y Santacreu (2014) y otros, sobre el mayor rechazo a los partidos tradicionales entre los jóvenes ante una situación de crisis y la búsqueda de mecanismos no convencionales de mediación de su voluntad política.

${ }^{5}$ Entre otras declaraciones, en noviembre de 2014 Pablo Iglesias asegura que «la política entre izquierda y derecha es una estafa» y que se trata «de oligarquía frente a ciudadanía». http:// www.lasexta.com/programas/sexta-columna/avances/pablo-iglesias-politica-izquierda-derechaestafa_2014110600308.html

EMPIRIA. Revista de Metodología de Ciencias Sociales. N. 38 septiembre-diciembre, 2017, pp. 151-176. ISSN: 1139-5737, DOI/empiria.38.2018.19715 
No sería, pues, "apatía o desinterés" por los asuntos políticos lo que motiva las tradicionales menores tasas de participación electoral entre los jóvenes, sino un mayor alejamiento de la política convencional. Su mayor búsqueda de vías no convencionales de expresión política habría propiciado un mayor abstencionismo, que disminuye ante la aparición de nuevas opciones políticas. Finalmente, la cuestión generacional es clave para entender el papel de las nuevas tecnologías, tanto para la dinamización descentralizada y la visibilización pública de los nuevos movimientos sociales (Romanos y Sádaba, 2015; Treré et al. 2017), como para la intensa experimentación tecnopolítica en el momento de emergencia de los nuevos partidos, desde Movimento 5 Stelle (Borreca, 2014) hasta el Partido X y Podemos (Toret, 2015; Romanos y Sádaba, 2015; Subirats, 2015). ${ }^{6}$

Resulta de interés el contraste de los datos obtenidos con las explicaciones centradas en las características del entorno político y económico del país (Müller-Rommel, 1998; 2002; Katz y Mair, 1995; 2009; Mair, 2001; Kitschelt, 1989) en el paso de los movimientos ecologistas a los partidos verdes. En particular, Müller-Rommel (1998) argumenta que los países con sistemas de partidos fuertemente polarizados son menos propensos a tener partidos verdes fuertes; si hay gran distancia ideológica entre los partidos mayoritarios, esta polarización domina el debate público y margina a los partidos minoritarios. En una línea similar, la cartelización de los grandes partidos (Mair, 2001) y la crisis de participación (Kitschelt, 1989) parecen facilitar la aparición de partidos outsiders o anti-establisment, como sucedió en los casos de los partidos verdes alemán y belga. Estas tres situaciones (escasa polarización, cartelización y crisis de participación) se cumplen, tanto en el momento en que aparece el $15 \mathrm{M}$ como Podemos. Asimismo, estas situaciones se reflejan en el marco comunicativo tanto del movimiento como, posteriormente, de Podemos, quienes se refieren a los partidos tradicionales como 'el bipartidismo', 'la vieja política', 'la casta' e incluso 'PPSOE' (representados en un solo bloque).

Los datos apuntan a que la convergencia de estas situaciones de crisis política tendría un efecto asimétrico en la dinámica electoral a lo largo del arco ideológico, mucho más intensamente de lo que ocurre en el apoyo a los movimientos de protesta. Así, los movimientos de protesta parecen obtener más fácilmente un apoyo transversal a lo largo del eje izquierda-derecha en una situación de descontento y erosión del apoyo político mientras que, en ese mismo contexto social, las nuevas formaciones políticas, al menos en España y en un primer momento, recibieron un apoyo electoral más fácilmente entre sectores ideológicos de izquierda que entre la derecha -como el caso de Vox, que no logró entrar en las instituciones europeas, nacionales o regionales-.

Una diferencia llamativa con lo señalado en el caso de los partidos verdes (Müller-Rommel, 1998) es que la aparición de Podemos se da en un contexto de crisis económica. En las elecciones al PE recibe parte de su apoyo de quienes tienen una mayor percepción de precarización (Fernández-Albertos, 2015). Esto

${ }^{6}$ Véase Robles et al. (2016) para un análisis de la brecha de participación política digital y de la influencia de la edad en dicha brecha.

EMPIRIA. Revista de Metodología de Ciencias Sociales. N. 38 septiembre-diciembre, 2017, pp. 151-176. ISSN: 1139-5737, DOI/empiria.38.2018.19715 
no encajaría fácilmente con la explicación post-materialista de Ronald Inglehart (1995; 1997). En la emergencia de Podemos, la insatisfacción política estaría íntimamente relacionada con la preocupación/indignación por la situación económica, particularmente entre los jóvenes. La emergencia de Podemos tiene, por tanto, similitudes y diferencias con el caso de los partidos verdes. Similitudes en torno a la capitalización de la desafección, la baja polarización y la cartelización de los partidos tradicionales, y diferencias en torno al contexto económicolaboral en el que aparecen.

Las similitudes con el éxito de partidos de carácter indigenistas en América Latina existen, aunque son también limitadas. En particular, el éxito de las movilizaciones del $15 \mathrm{M}$-por su dimensión cuantitativa- parece haber aportado el "momento de euforia", de optimismo de que un cambio es posible, que apunta Van Cott (2005) para los casos latinoamericanos. Tras la rápida extensión de las movilizaciones por todo el país en 2011 y el amplio apoyo reflejado en las encuestas de opinión pública, muchos electores y activistas pueden vislumbrar que "sí se puede" extender las protestas al terreno electoral. Desde la óptica de su función para la creación de nuevos partidos, las movilizaciones de los indignados sirvieron para visibilizar un disenso consensual en torno a la cultura política del bipartidismo, permitiendo la ruptura de un eventual sesgo de ignorancia pluralista en torno a él -la creencia de que únicamente una minoría confronta el consenso bipartidista, cuando en realidad el apoyo en la calle y en las encuestas era mayoritario-. Este proceso cambia la percepción de la situación política y, por tanto, la disposición para la acción en el campo político, también a través del voto.

Tras su aparición exitosa en las elecciones al PE, la intención de voto a Podemos aumentó considerablemente en pocos meses -pasó de obtener un 3,52\% del voto sobre censo electoral en las elecciones al PE de 2014 a aglutinar el 17,6 $\%$ de la intención de voto cinco meses después-. En este crecimiento, la composición de su electorado se transformó, lo que hace necesario distinguir entre la primera fase de cristalización electoral, la emergencia del partido ante sus primeras elecciones en mayo de 2014, y una posterior fase de crecimiento, en la que esta cristalización electoral parece ampliarse entre colectivos de características diferentes a los de la fase de emergencia. En esta primera fase de crecimiento, entre mayo y octubre de 2014, Podemos presenta una mayor capacidad de atraer a un electorado no activista, tanto entre los abstencionistas como entre los votantes de partidos tradicionales: el índice de cristalización se duplica entre los votantes del PSOE, IU y PP, y se triplica entre los abstencionistas. 


\section{CONCLUSIONES}

El creciente interés académico por la institucionalización del ciclo de protestas del 15M (Andretta y Della Porta, 2015; Martín, 2015; Tormeya y Feenstra, 2015; Calle, 2015; Subirats, 2015) contrasta con un déficit de datos cuantitativos sobre esta cuestión. Con el fin de superar la carencia de datos integrados sobre el apoyo al $15 \mathrm{M}$ y de apoyo electoral a Podemos, hemos desarrollado el cálculo de un índice de cristalización electoral (Ic). Mediante esta magnitud cuantificamos el comportamiento de los diferentes sectores de población y analizamos qué factores acompañan, en mayor medida, el paso de la simpatía por un movimiento social al voto por un nuevo partido.

Los resultados muestran, en primer lugar, que los diferentes sectores sociales presentan diferentes índices de cristalización electoral. Es decir, la traducción electoral del apoyo al $15 \mathrm{M}$ se produjo de manera heterogénea entre colectivos. En particular, existen sectores sociales donde un elevado nivel de apoyo al $15 \mathrm{M}$ apenas cristalizó en voto a la nueva formación política en sus primeras elecciones. La mayor discriminación se observa en torno a la ideología. Así, constatamos que la simpatía por el movimiento de protesta cristalizó en voto a Podemos cinco veces más entre los votantes del IU (Ic=25.6) que entre los votantes del PP $(I c=5.6)$, y casi tres veces más entre los votantes del PSOE (Ic=14.0). También es especialmente bajo el índice de cristalización electoral entre los mayores de 64 años, entre los jubilados y pensionistas y las amas de casa, entre quienes tienen un nivel educativo bajo y entre aquellas personas que viven en municipios con menos de 10.000 habitantes. A pesar de que el 15M logró un apoyo mayoritario entre cada uno de estos segmentos de población, la nueva formación política no consiguió el apoyo electoral entre los propios simpatizantes del 15M que forman parte de estos sectores de población -sectores que, por otro lado, responden tradicionalmente a patrones de voto más conservadores-.

El reciente estudio de Portos y Masullo (2017) muestra que los factores de movilización en el 15M fueron principalmente la ideología y el activismo previo. Es entre estos mismos sectores en los que el nuevo partido habría obtenido su primera base social electoral. Esta observación apunta a una continuidad entre la protesta y el voto a la nueva formación. Es decir, podemos entender el voto a Podemos en las elecciones al PE como una forma de extender el posicionamiento político crítico desarrollado en ciclo de protesta más allá de su finalización. Esta continuidad con la protesta caracteriza la aparición del nuevo partido, tanto en la base social de su primer electorado, como hemos visto, así como en lo que se refiere a su aspecto narrativo-simbólico. La caracterización del voto a Podemos como un "voto protesta" en el sentido planteado por Van der Eijk et al. (1996) debería ser matizada, ya que el primer votante de Podemos sí presenta un voto ideológico -vinculado a los planteamientos $15 \mathrm{M}-$. Más que un voto de protesta estaríamos ante un voto de la protesta, llevando la ideología de la protesta al campo electoral -o al menos, este sería el contexto en el que presumiblemente decide su voto su base electoral inicial-.

Finalmente, constatamos que la crisis política tendría un efecto asimétrico 
en la dinámica electoral a lo largo del arco ideológico, de un modo mucho más intenso de lo que ocurre en el apoyo a los movimientos de protesta. La ideología se presenta como el principal regulador del efecto asimétrico de la crisis política. Así, en un primer momento, la erosión del apoyo político entre 2008 y 2011 presenta un alto nivel de transversalidad ideológica (Lobera y Ferrándiz, 2013). Durante las movilizaciones de protesta en 2011, la ideología diferencia, principalmente, a quienes participan más activamente en ellas (Portos y Masullo, 2017) pero tiene un efecto más matizado entre quienes apoyan sus reivindicaciones (Sampedro y Lobera, 2014). Ante la emergencia de un partido-extensión del ciclo de protesta (al menos, así podríamos caracterizar la dinámica electoral de Podemos en las elecciones de EP de 2014), la ideología produce un fuerte efecto modulador de la cristalización electoral del apoyo al movimiento, como hemos visto en este trabajo. Finalmente, en las elecciones generales de 2015, se constata que la ideología y la desafección política son los principales factores explicativos del voto a Podemos (Orriols y Cordero, 2016). La crisis política y la ideología están, pues, vinculadas a lo largo del ciclo descrito, que va desde las opiniones cotidianas (incluyendo su medición en las encuestas), pasando por la movilización, hasta su extensión en el campo electoral. Así, de manera consistente a lo avanzado en $2013,{ }^{7}$ la crisis política tendría unos impactos electorales asimétricos a lo largo del arco ideológico, acentuando su efecto electoral entre los partidos de la izquierda y moderándolo entre los sectores más conservadores.

\section{REFERENCIAS}

ALCÁNTARA SÁEZ, M. y FREIDENBERG, F. (2001): «Los partidos políticos en América Latina”, América Latina Hoy, 27, pp. 17-35.

ANDRETTA, M. y DELLA PORTA. D. (2015): "Contentious precarious generation in anti-austerity movements in Spain and Italy", OBETS: Revista de Ciencias Sociales, 10(1), pp. 37-66.

ANDUIZA, E., MARTÍN, I. y MATEOS, A. (2012): "Las consecuencias electorales del $15 \mathrm{M}$ en las elecciones generales de 2011", Arbor. Ciencia, Pensamiento y Cultura, 188 (756).

BORRECA, R. (2014): "Political Crisis in Greece and Italy: a comparative analysis of SYRIZA and 5 Stars Movement", en PSA 64th Conference.

BOSCO, A. y VERNEY, S. (2012): "Electoral Epidemic: The Political Cost of Economic Crisis in Southern Europe, 2010-11", South European Society and Politics, 17(2), pp. 129-154.

CALLE, A. (2015): "Podemos y el auge municipalista. sobre partidos-ciudadanía y vieja política", EMPIRIA. Revista de Metodología de Ciencias Sociales, 32, septiembrediciembre, pp. 169-190.

CALVO, K. y ÁLVAREZ, I. (2015): "Limitaciones y exclusiones en la institucionalización de la indignación: del 15M a Podemos", Revista Española de Sociología, 24, pp. 119-125.

${ }^{7}$ Para una explicación más detallada del efecto asimétrico de la crisis política en la dinámica electoral, véase Lobera y Ferrándiz (2013).

EMPIRIA. Revista de Metodología de Ciencias Sociales. N. 38 septiembre-diciembre, 2017, pp. 151-176. ISSN: 1139-5737, DOI/empiria.38.2018.19715 
CANTON, S. (1995): "Partidos Políticos en las Américas: Desafíos y Estrategias", Washington DC, National Democratic Institute.

CORDERO, G. Y MONTERO, J. R. (2015): "Against Bipartyism, Towards Dealignment? The 2014 European Election in Spain”, South European Society and Politics, 20(3), pp. 357-379.

DE SOUSA SANTOS, B. (2014): "La ola Podemos", Página 12, disponible en http://www.pagina12.com.ar/diario/elmundo/4-260444-2014-11-23.html

VAN DER EIJK, C., FRANKLIN, M. y MARSH, M. (1996), "What voters teach us about European elections/what European elections teach us about voters", Electoral Studies, 15, pp. 149-166.

FEENSTRA, R. A., TORMEY, S., CASERO-RIPOLLÉS, A., Y KEANE, J. (2017): "Refiguring democracy: The Spanish political laboratory", New York: Routledge.

FERNÁNDEZ-ALBERTOS, J. (2015): "Los votantes de Podemos. Del partido de los indignados al partido de los excluidos", Madrid, Los libros de la Catarata.

FERNÁNDEZ-SAVATER, A. (2012): "El nacimiento de un nuevo poder social", Hispanic Review, 80, pp. 667-682.

FLESHER FOMINAYA, C. (2014): “Debunking Spontaneity: Spain's 15-M/Indignados as Autonomous Movement", Social Movement Studies, 14(2), pp. 142-163.

FRANCÉS, F. J. y SANTACREU, O. (2014): “Crisis política y juventud en España: el declive del bipartidismo electoral”, SocietàMutamentoPolitica, 5, pp.107-128.

INGLEHART, R. (1995): "Public Support for Environmental Protection: Objective Problems and Subjective Values in 43 Societies", PS: Political Science and Politics, 28, pp. 57-72.

INGLEHART, R. (1997): "Modernization and Postmodernization: Cultural, Economic and Political Change in 43 Societies", Princeton NJ, Princeton University Press.

JIMÉNEZ, M. (2011): “iInfluyó el 15M en las elecciones municipales?”, Zoom político, 4, Madrid, Fundación Alternativas.

KASSE, M. (2007): "Perspectives on Political Participation", The Oxford Handbook of Political Behaviour, New York, Oxford University Press.

KATZ, R. S. y MAIR, P. (1995): "Changing models of party organization and party democracy the emergence of the cartel party", Party politics, 1, pp. 5-28.

KATZ, R. S. y MAIR, P. (2009): "The cartel party thesis: A restatement", Perspectives on politics, 7, pp. 753-766.

KITSCHELT, H. (2006): "Movement Parties", en Handbook of Party Politics, Londres, Sage, pp. 278-290.

LOBERA, J. (2015): "De movimientos a partidos. La cristalización electoral de la protesta", en Revista Española de Sociología, 24, pp. 97-105.

LOBERA, J. y FERRÁNDIZ, J. P. (2013): "El peso de la desconfianza política en la dinámica electoral en España", en Partidos, medios y electores en procesos de cambio. Las Elecciones Generales españolas de 2011, Valencia, Editorial Tirant Lo Blanch, pp. 41-65.

LOBERA, J. Y PAREJO, D. (2017): "Streets and institutions? The electoral extension of social movements and its tensions", en Routledge Handbook of Radical Politics, eds Uri Gordon and Ruth Kinna, London: Routledge (en prensa).

LOBERA, J. y SAMPEDRO, V. (2014): "La transversalidad del 15M entre la ciudadanía", en 15MP2P. Una mirada transdisciplinar del 15M, Barcelona, UOC, pp. 470489.

MCADAM, D. y TARROW, S. (2011). In Funes, MJ. (2011) A propósito de Tilly. Conflicto, poder y acción colectiva. Centro de Investigaciones Sociológicas. 
MARTíNEZ, G. (2012): “CT o la Cultura de la Transición: crítica a 35 años de cultura española", Barcelona, Debolsillo.

MAIR, P. (2001): "The Green Challenge and Political Competition: How Typical is the German Experience?" German Politics, 10, pp. 99-116.

MARTÍN, I. (2015): "Tres modelos de partido-movimiento", Revista Española de Sociología, 24, pp. 111-118.

MORENO-CABALLUD, L. (2013): "Desbordamientos culturales en torno al 15M", en Teknokultura, 10, pp. 101-130.

MÜLLER-ROMMEL, F. (1998): "Explaining the Electoral Success of Green Parties: A Cross-National Analysis", Environmental Politics, 7, pp. 145-154.

MÜLLER-ROMMEL, F. (2002): “The Lifespan and the Political Performance of Green Parties in Western Europe", Environmental Politics, 11, pp. 1-16.

MURO, D. Y VIDAL G. (2016): "Political mistrust in southern Europesince the Great Recession", Mediterranean Politics, 0(0) 1-21.

ORRIOLS, L. y CORDERO, G. (2016): "The Breakdown of the Spanish

Two-Party System: The Upsurge of Podemos and Ciudadanos in the 2015 General Election", South European Society and Politics, pp. 1-24.

PORTOS, M. y MASULLO, J. (2017): "Voicing Outrage Unevenly. Democratic Dissatisfaction, Non-participation and Frequencies of Participation in the 15-M Campaign", Mobilization: An International Quarterly 22(2), pp. 201-222.

RODON, T. y HIERRO, M. J. (2016): "Podemos and Ciudadanos Shake up the Spanish Party System: The 2015 Local and Regional Elections", South European Society and Politics, 21(3), pp. 339-357.

ROBERTS, K. M. (1998): "Deepening Democracy? The Modern Left and Social Movements in Chile and Peru", Stanford, Stanford University Press.

ROBLES, J. M., ANTINO, M., DE MARCO, S. y LOBERA, J. (2016): "La nueva frontera de la desigualdad digital: la brecha participativa", Revista Española de Investigaciones Sociológicas, 156, pp. 97-116.

ROMANOS, E. y SÁDABA, I. (2015): "La evolución de los marcos (tecno) discursivos del movimiento 15M y sus consecuencias", Empiria, 32, pp. 15-36.

ROMANOS, E. y SÁDABA, I. (2016): "From the Street to Institutions through the App: Digitally Enabled Political Outcomes of the Spanish Indignados Movement", Revista Internacional de Sociología 74 (4): e048. doi: http://dx.doi.org/10.3989/ ris.2016.74.4.048

SAMPEDRO, V. y LOBERA, J. (2014): “The Spanish 15M movement: a consensual dissent?", Journal of Spanish Cultural Studies, 15, pp. 68-80.

SÁNCHEZ, O. (2008): "Transformation and Decay: The De-Institutionalisation of Party Systems in South America", Third World Quarterly, 29, pp. 315-337.

SCHMITTER, P. (2006): "Interests", European University Institute, disponible en http:// www.eui.eu/Documents/DepartmentsCentres/SPS/Profiles/Schmitter/PCSInterestsDec06.pdf

SUBIRATS, J. (2015): “Todo se mueve. Acción colectiva, acción conectiva. Movimientos, partidos e instituciones", Revista Española de Sociología, 24, 127-134.

TILLY, C. (2004): "Contention and democracy in Europe, 1650-2000", Cambridge, Cambridge University Press.

TORET, J. (2015): "Una mirada tecnopolítica al primer año de Podemos. Seis hipótesis”, Teknokultura, 12, pp. 121-135.

TORMEYA, S. y FEENSTRA, R. A. (2015): "Reinventing the political party in Spain: the case of $15 \mathrm{M}$ and the Spanish mobilisations", Policy Studies, 36(6), pp. 590-606. 
TRERÉ, E, JEPPESEN, S. Y MATTONI, A. (2017): “Comparing Digital Protest Media Imaginaries: Anti-austerity Movements in Spain, Italy \& Greece", tripleC: Communication, Capitalism \& Critique, 15(2), pp. 390-542.

TSAKATIKA, M. (2015), "Syriza y la izquierda en la política griega", en Grecia: Aspectos políticos y jurídico-económicos de la crisis, Madrid, Centro de Estudios Políticos y Constitucionales, pp. 79-98.

VAN COTT, D. L. (2005): "From Movements to Parties in Latin America. The Evolution of Ethnic Parties", Cambridge, Cambridge University Press.

VERNEY, S. (2014), “'Broken and Can't be Fixed': The Impact of the Economic Crisis on the Greek Party System”, The International Spectator, 49, pp. 18-35. . 